\title{
Post-transplant malignancies in alcoholic liver disease
}

\author{
Akash Singh, Arka De, Virendra Singh \\ Department of Hepatology, Post Graduate Institute of Medical Education and Research, Chandigarh, India \\ Contributions: (I) Conception and design: A Singh; (II) Administrative support: None; (III) Provision of study materials or patients: None; (IV) \\ Collection and assembly of data: None; (V) Data analysis and interpretation: None; (VI) Manuscript writing: All authors; (VII) Final approval of \\ manuscript: All authors. \\ Correspondence to: Virendra Singh, MD, DM, FASGE. Professor, Department of Hepatology, Post Graduate Institute of Medical Education and \\ Research, Chandigarh, India. Email: virendrasingh100@hotmail.com.
}

\begin{abstract}
Post-transplant malignancy is emerging as an important cause of mortality in patients with cirrhosis undergoing liver transplant (LT). However, establishing the exact relationship between the two needs further evaluation. It has been observed that approximately $30 \%$ deaths after 10 years of hepatic transplantation occur due to de novo malignancies. Various known risk factors include immunosuppression, age of patient, alcoholic liver disease (ALD) or primary sclerosing cholangitis, smoking, and oncogenic viral infections. There is scanty literature on the post-transplant malignancy risk in patients with alcoholic cirrhosis. The current evidence suggests a particularly increased risk of oropharyngeal and lung cancers in patients transplanted for ALD. Abstinence from alcohol, smoking and other tobacco-containing products along with optimization of immunosuppression are paramount for decreasing the risk of post-transplant malignancies.
\end{abstract}

Keywords: Alcoholic cirrhosis; hepatic transplantation; cancer; de novo malignancy

Received: 20 July 2019; Accepted: 21 November 2019; Published: 05 April 2020.

doi: $10.21037 / \operatorname{tgh} .2019 .11 .18$

View this article at: http://dx.doi.org/10.21037/tgh.2019.11.18

\section{Introduction}

Liver transplantation (LT) is the treatment of choice for patients with end-stage liver disease. Post-transplantation malignancy is becoming a major problem with the increased longevity of these patients. Professor Thomas Starzl and co-workers in 1968 envisioned increased incidence of de novo malignancies in immunocompromised patients which was later confirmed by others (1-3). The incidence of posttransplant de novo malignancies ranges from $3 \%$ to $26 \%$ (4-7). This variation may be due to the difference in the duration of follow-up, geographic variations and different ways of reporting. Various studies have reported a 2-times higher risk of solid organ tumors and a $\geq 30$-times higher risk of lymphoproliferative neoplasia as compared to general population (8-11). The mortality due to de novo malignancies account for $0.6 \%$ and $8.0 \%$ in this population $(12,13)$. Various studies have demonstrated higher incidence of $d e$ novo malignancies in alcoholic as compared to non-alcoholic
LT recipients (4,14-18). Immunosuppression, hepatitis C virus (HCV) infection, smoking, alcoholic cirrhosis, and sun exposure have been shown to be important risk factors for the development of post-transplant de novo malignancies in a systematic review $(19,20)$. However, there is paucity of literature regarding these factors. In the present review, we will discuss post-transplant malignancies, summarize the evidence behind increased risk of malignancies after transplantation for alcoholic liver disease (ALD) and outline suggested screening and preventive strategies.

\section{Post-transplant malignancies}

Neoplasms developing post-transplant are called de novo malignancies. About 1\% patients develop solid organ malignancies annually, with an overall incidence of 3-15\% after LT $(14,19)$. Post-transplant lymphoproliferative disorders (PTLDs) occur in about $20 \%$ of cases (21). Incidences of various neoplasms in these patients have been 
reported as follows: Kaposi's sarcoma (17\%), head and neck cancer (17\%), esophageal carcinoma (12\%), lung cancer $(10 \%)$, gastric adenocarcinoma (7\%), melanoma (5\%), colorectal cancer $(5 \%)$, cervical cancer $(5 \%)$, and breast cancer (2\%) (21).

Several risk factors linked to the development of cancer in the general population also have relevance in the posttransplant setting. These include the consumption of alcohol, smoking, immunosuppression, recipient age and oncogenic viral infections like HCV and Epstein-Barr virus (EBV). The use of immunosuppressants like OKT3, anti-thymocyte globulin and calcineurin inhibitors like tacrolimus are important risk factors for the development of de novo malignancies post-transplant $(22,23)$. However, mTOR inhibitors like sirolimus have been associated with a decreased risk of malignancies $(24,25)$. Other risk factors identified by a recent systematic review include the use of tobacco and alcohol, older age and pre-malignant conditions as the important risk factors for the development of post-transplant malignancies (20).

\section{Skin cancer}

Squamous cell carcinoma is the most common dermatological cancer in the post-transplant setting in contrast to basal cell carcinoma which is more common in the general population. Various factors like higher age, increased intensity and prolonged use of immunosuppressive therapy, human papillomavirus infection, prolonged exposure to ultraviolet rays, history of actinic keratosis, CD4 lymphocytopenia, blue or hazel eyes have been implicated (26,27). Patients undergoing LT for primary sclerosing cholangitis and alcoholic cirrhosis are at a higher risk of developing skin cancer in comparison to patients of other etiologies $(4,15,28,29)$.

\section{PTLD}

PTLD has been reported to be the second most common malignancy in LT recipients being $0.9 \%$ to $2.6 \%$ in comparison to $0.03 \%$ in general population $(6,16)$. EBV and HCV have been implicated in the development of PTLD in various studies (22,30-33). The incidence of PTLD is not specifically higher in patients transplanted for ALD (17). However, the mortality after development of PTLD was higher in patients transplanted for ALD as compared to other etiologies (34-37).

\section{Lung cancer}

LT recipients have a higher risk of lung cancer $(0 \%$ to $1.2 \%)$ than that of the general population $(0.06 \%)(4,16)$. The mean time to diagnosis of lung cancer ranges from 42 to 50 months (38-41). The diagnosis of lung cancer is often made in advanced stages and carries a poor prognosis. Etiologically, patients with alcoholic cirrhosis undergoing LT have a 2 to 4 times higher risk of lung cancer (42). The risk is further compounded by the fact that many patients who drink alcohol are also smokers.

\section{Head and neck cancers}

Head and neck cancers occur more frequently $(0.1-2 \%)$ after LT than in the general population $(16,41)$. The mean time to diagnosis varies from 34.3 to 61.2 months $(4,38,40,43,44)$. Patients with alcoholic cirrhosis undergoing LT are more prone to develop oropharyngeal cancer as compared to patients transplanted for other etiologies. In fact, in one single-center study, no case of oropharyngeal cancer was reported in the absence of history of alcohol intake or cigarette smoking (45).

\section{Esophageal and gastric cancer}

Gastric and esophageal cancers are infrequent in LT recipients but their incidence is higher than the general population (46). In a study of 313 LT recipients over a span of 15 years, 40 patients developed de novo malignancy of which $12 \%$ had esophageal cancer (21). In a German study of 1,926 LT recipients, 9 patients developed esophageal cancer and 1 developed cancer of the cardia (47). In this study, squamous cell carcinoma was seen in $7 / 10$ and adenocarcinoma in $3 / 10$ patients and 9 of these 10 patients had undergone LT for alcoholic cirrhosis (47). Increased risk of esophageal cancer in patients transplanted for ALD has also been in other studies $(4,22,48)$.

\section{Colorectal cancer}

Colorectal cancer occurs more frequently in the posttransplant setting $(0-0.6 \%)$ compared to the general population $(0.05 \%)(6,39,40,49)$. A study from the Netherlands showed a 12 times higher risk of colorectal neoplasia after LT (19). This high incidence may be attributed to the presence of ulcerative colitis in patients with primary sclerosing cholangitis who underwent 
Table 1 Incidence of de novo malignancies post-transplant in alcoholic vs. non-alcoholic groups

\begin{tabular}{lccc}
\hline \multirow{2}{*}{ Malignancies } & \multicolumn{2}{c}{ Incidence } & Reference number \\
\cline { 2 - 3 } Skin cancer & Non-alcoholic & Alcoholic & $(4,15,28,29)$ \\
PTLDs & Not increased & Increased & $(17)[$ high mortality: (34-37)] \\
Lung cancer & Not increased & Not increased & $(42,53-56)$ \\
Head and neck cancer & Not increased & Increased & $(17,22,43,45,53,54)$ \\
Esophageal and gastric & Not increased & Increased & $(4,22,47,48,53)$ \\
Colorectal & Not increased & Increased & $(17)$ \\
Genitourinary & Not increased & Not increased & Insufficient data \\
Gynecological & & Insufficient data & \\
\hline
\end{tabular}

PTLD, post-transplant lymphoproliferative disorder.

transplant (50-52). However, the risk of colorectal cancer does not appear to be increased in transplant recipients of alcoholic cirrhosis (17).

\section{Genitourinary cancer}

In LT recipients, the incidence of prostate cancer is not increased and that of bladder and renal cancer is higher than that of the general population $(4,22,38)$. The average time taken to diagnose these genitourinary neoplasms varies between 20 to 55.3 months $(4,38)$. There is insufficient data to draw conclusions regarding alcoholic cirrhosis.

\section{Gynecological cancer}

The incidence of cervical and ovarian cancers is higher in LT recipients in comparison to the general population $(4,39,40)$. On the other hand, the risk of breast cancer does not appear to be higher in LT recipients as compared to the general population (12). However, there is limited data to comment on the incidence of malignancies in the specific subgroup of patients transplanted for ALD.

\section{ALD and post-transplant malignancy}

There is limited data on the risk of post-transplant malignancies in alcoholic cirrhosis vis-à-vis patients transplanted for non-alcohol related etiologies (Table 1). In a multicentric, US study including almost 800 recipients, alcoholic cirrhosis was an independent risk factor for the development of post-transplant malignancy with a hazard ratio of 2.4 (14). The increased risk of de novo malignancies in patients transplanted for ALD was confirmed in a recently published French National follow-up study of 11,226 recipients of whom $36 \%$ had been transplanted for ALD (53). This increased risk was present in both genders. A significantly increased risk of neoplasms of the larynx, esophagus, lip-mouth-pharynx, and lung was observed in patients who had been transplanted for ALD as compared to non-alcohol related etiologies (53).

The available data suggests that risk of lung cancers, upper respiratory tract and oropharynx is particularly increased after transplantation for ALD (17,22,43,53-56). In a Spanish study, upper respiratory tract and lung cancers were seen in $8 \%$ patients transplanted for alcoholic cirrhosis as compared to $0.8 \%$ of patients transplanted for nonalcohol related etiologies (55). In a study of 700 recipients, post-transplant lung cancer was detected in $17(2 \%)$ patients of which $12(1.7 \%)$ had been transplanted for alcoholic cirrhosis (56). Jiménez et al. also demonstrated higher rates of lung cancer in those who underwent LT for alcoholic cirrhosis than those transplanted for other etiologies (4.3\% vs. $0.7 \%, \mathrm{P}<0.001)$. Almost $75 \%$ of the lung cancer patients in their study used to drink alcohol heavily and they were also intermediate or heavy smokers (54). There is some evidence regarding the use of annual chest $\mathrm{X}$-rays or computed tomography (CT) scans as surveillance in these patients, particularly in smokers (57-59).

The risk of oropharyngeal cancers is also increased in patients transplanted for ALD in comparison to other etiologies. Duvoux et al. reported a higher incidence in patients transplanted for alcoholic etiology than nonalcoholic one $(16.7 \%$ vs. $0 \%$; $\mathrm{P}=0.001)$ (43). The risk of post-transplant oropharyngeal cancer was reported to 
Table 2 Suggested screening and preventive strategies for post-transplant malignancies in patients transplanted for alcoholic cirrhosis

\begin{tabular}{|c|c|}
\hline Cancer type & Suggested screening and preventive strategy \\
\hline \multirow{2}{*}{ Skin cancer } & Reduced sun-exposure (caps, long sleeve clothing and trousers) \\
\hline & Sun-screens \\
\hline Lung cancer & $\begin{array}{l}\text { Annual chest } X \text {-rays or low-dose CT chest (particularly in smokers with cumulative } \\
\text { pack years of }>10 \text { ) }\end{array}$ \\
\hline \multirow[t]{2}{*}{ Oropharyngeal and laryngeal cancers } & Annual otolaryngology evaluation \\
\hline & Stop smoking and tobacco products \\
\hline PTLDs & EBV status prior to transplant \\
\hline Cervical cancer (in females) & Cervical cytology as per local guidelines for general population \\
\hline Prostate cancer (in males) & $\begin{array}{l}\text { Annual prostate specific antigen beginning at } 50 \text { years of age or as per local } \\
\text { guidelines for general population }\end{array}$ \\
\hline
\end{tabular}

CT, computed tomography; PTLD, post-transplant lymphoproliferative disorder; EBV, Epstein-Barr virus.

be 25 times higher in alcoholic cirrhosis than in other etiologies by Jain et al. (17). In another Spanish study, alcoholic cirrhosis emerged as an independent risk factor for de novo malignancies post-transplant with a relative risk of 2 (22). Forty-four percent of those who developed de novo malignancies had alcoholic cirrhosis. The incidence of de novo malignancies post-transplant was $9 \%$ in alcoholic cirrhosis as compared to $3 \%$ in non-alcohol related etiologies. Majority of the neoplasms detected were classified oropharyngeal tumors and based on this the authors recommended annual screening for oropharyngeal malignancies in patients transplanted for ALD irrespective of abstinence (22).

\section{Prevention}

As post-LT patients are at a higher risk of developing malignancies, there is a need of intensive screening protocols. Finkenstedt et al. compared an "intensified" surveillance protocol comprising of yearly thoracic and abdominal CT scan, pap smear, mammography, urological and dermatologic evaluation along with colonoscopy every 5 -year with a "historical" protocol of annual chest X-rays and ultrasonography (USG) of the abdomen (57). The "intensified" protocol resulted in fewer malignancies being diagnosed at an advanced stage ( $46 \% v s .75 \%$ ) and an increased survival in non-dermatologic malignancies (3.3 years $v s$. $75 \%$ ) as compared to the "historical" protocol (57). Herrero et al. also reported improved survival after cancer diagnosis using a multipronged surveillance protocol consisting of urine examination, chest X-ray, and abdominal-USG every 6 months in the $1^{\text {st }}$ year and annually thereafter; mammography every 2 years and colonoscopy every 7-10 years. Additionally, annual otolaryngologic examinations and low-dose CT thorax were also performed in smokers (59). There have been concerns about the risk of radiation in these patients who have probably undergone multiple CT scans in the pre-transplant period. Low-dose CT protocols can help attenuate the radiation risk entailed and screening with yearly low-dose chest CT scans in smokers (cumulative pack years $>10$ ) helped diagnose posttransplant lung cancer in early stages in $12 \%$ patients (58). A suggested screening schedule is given in Table 2. The risk of colorectal and breast cancer does not seem to be significantly greater than that in the general population. Thus, screening for these conditions as per local guidelines for the general population should suffice (60). Smoking cessation and strict avoidance of tobacco-containing products is the 
most important preventive measure as emphasized in the AASLD guidelines (61). Immunosuppression should be optimized and calcineurin inhibitors in particular should be minimized.

\section{Conclusions}

In conclusion, there is a high risk of post-transplant malignancies in LT recipients. The major factor appears to be immunosuppression. The incidence of certain malignancies including oropharyngeal and lung cancers are particularly increased in patients undergoing transplant for alcoholic cirrhosis. There is a need for cost-effective protocols for prevention of neoplasms in these patients.

\section{Acknowledgments}

None

\section{Footnote}

Conflicts of Interest: The authors have no conflicts of interest to declare.

Ethical Statement: The authors are accountable for all aspects of the work in ensuring that questions related to the accuracy or integrity of any part of the work are appropriately investigated and resolved.

\section{References}

1. McKhann CF. Primary malignancy in patients undergoing immunosuppression for renal transplantation. Transplantation 1969;8:209-12.

2. Penn I. Incidence and treatment of neoplasia after transplantation. J Heart Lung Transplant 1993;12:S328-36.

3. Penn I, Hammond W, Brettschneider L, et al. Malignant lymphomas in transplantation patients. Transplant Proc 1969;1:106-12.

4. Saigal S, Norris S, Muiesan P, et al. Evidence of differential risk for posttransplantation malignancy based on pretransplantation cause in patients undergoing liver transplantation. Liver Transpl 2002;8:482-7.

5. Herrero JI, Lorenzo M, Quiroga J, et al. De novo neoplasia after liver transplantation: an analysis of risk factors and influence on survival. Liver Transpl 2005;11:89-97.

6. Yao FY, Gautam M, Palese C, et al. De novo malignancies following liver transplantation: a case-control study with long-term follow-up. Clin Transplant 2006;20:617-23.

7. Kelly DM, Emre S, Guy SR, et al. Liver transplant recipients are not at increased risk for nonlymphoid solid organ tumors. Cancer 1998;83:1237-43.

8. Taylor AL, Marcus R, Bradley JA. Post-transplant lymphoproliferative disorders (PTLD) after solid organ transplantation. Crit Rev Oncol Hematol 2005;56:155-67.

9. Aberg F, Pukkala E, Höckerstedt K, et al. Risk of malignant neoplasms after liver transplantation: a population-based study. Liver Transpl 2008;14:1428-36.

10. Engels EA, Pfeiffer RM, Fraumeni JF Jr, et al. Spectrum of cancer risk among US solid organ transplant recipients. JAMA 2011;306:1891-901.

11. Collett D, Mumford L, Banner NR, et al. Comparison of the incidence of malignancy in recipients of different types of organ: a UK registry audit. Am J Transplant 2010;10:1889-96.

12. Chandok N, Watt KD. Burden of de novo malignancy in the liver transplant recipient. Liver Transpl 2012;18:1277-89.

13. Sanchez W, Talwalkar JA, Gores GJ. "Will all liver transplantation patients eventually die from cancer?". J Hepatol 2006;44:13-8.

14. Watt KD, Pedersen RA, Kremers WK, et al. Long-term probability of and mortality from de novo malignancy after liver transplantation. Gastroenterology 2009;137:2010-7.

15. Jiménez-Romero C, Manrique Municio A, Marqués Medina E, et al. Incidence of de novo nonmelanoma skin tumors after liver transplantation for alcoholic and nonalcoholic liver diseases. Transplant Proc 2006;38:2505-7.

16. Jiménez C, Rodríguez D, Marqués E, et al. De novo tumors after orthotopic liver transplantation. Transplant Proc 2002;34:297-8.

17. Jain A, DiMartini A, Kashyap R, et al. Long-term followup after liver transplantation for alcoholic liver disease under tacrolimus. Transplantation 2000;70:1335-42.

18. Bellamy CO, DiMartini AM, Ruppert K, et al. Liver transplantation for alcoholic cirrhosis: long term followup and impact of disease recurrence. Transplantation 2001;72:619-26.

19. Haagsma EB, Hagens VE, Schaapveld M, et al. Increased cancer risk after liver transplantation: a population-based study. J Hepatol 2001;34:84-91.

20. Chak E, Saab S. Risk factors and incidence of de novo malignancy in liver transplant recipients: a systematic review. Liver Int 2010;30:1247-58. 
21. Baccarani U, Adani GL, Serraino D, et al. De novo tumors are a major cause of late mortality after orthotopic liver transplantation. Transplant Proc 2009;41:1303-5.

22. Benlloch S, Berenguer M, Prieto M, et al. De novo internal neoplasms after liver transplantation: increased risk and aggressive behavior in recent years? Am J Transplant 2004;4:596-604.

23. Gutierrez-Dalmau A, Campistol JM. Immunosuppressive therapy and malignancy in organ transplant recipients. Drugs 2007;67:1167-98.

24. Neuhaus P, Klupp J, Langrehr JM. mTOR inhibitors: an overview. Liver Transpl 2001;7:473-84.

25. Mathew T, Kreis H, Friend P. Two-year incidence of malignancy in sirolimus-treated renal transplant recipients: results from five multicenter studies. Clin Transplant 2004;18:446-9.

26. Berg D, Otley CC. Skin cancer in organ transplant recipients: epidemiology, pathogenesis, and management. J Am Acad Dermatol 2002;47:1-17; quiz 18-20.

27. Carroll RP, Ramsay HM, Fryer AA, et al. Incidence and prediction of nonmelanoma skin cancer post-renal transplantation: a prospective study in Queensland, Australia. Am J Kidney Dis 2003;41:676-83.

28. Otley CC, Cherikh WS, Salasche SJ, et al. Skin cancer in organ transplant recipients: effect of pretransplant endorgan disease. J Am Acad Dermatol 2005;53:783-90.

29. Mithoefer AB, Supran S, Freeman RB. Risk factors associated with the development of skin cancer after liver transplantation. Liver Transpl 2002;8:939-44.

30. Shroff R, Rees L. The post-transplant lymphoproliferative disorder-a literature review. Pediatr Nephrol 2004;19:369-77.

31. Dierickx D, Tousseyn T, De Wolf-Peeters C, et al. Management of posttransplant lymphoproliferative disorders following solid organ transplant: an update. Leuk Lymphoma 2011;52:950-61.

32. Duvoux C, Pageaux GP, Vanlemmens C, et al. Risk factors for lymphoproliferative disorders after liver transplantation in adults: an analysis of 480 patients. Transplantation 2002;74:1103-9.

33. McLaughlin K, Wajstaub S, Marotta P, et al. Increased risk for posttransplant lymphoproliferative disease in recipients of liver transplants with hepatitis C. Liver Transpl 2000;6:570-4.

34. Van Thiel DH, Bonet H, Gavaler J, et al. Effect of alcohol use on allograft rejection rates after liver transplantation for alcoholic liver disease. Alcohol Clin Exp Res 1995;19:1151-5.
35. Lucey MR. Liver transplantation for alcoholic liver disease. Nat Rev Gastroenterol Hepatol 2014;11:300-7.

36. Pereira SP, Williams R. Liver transplantation for alcoholic liver disease at King's College Hospital: survival and quality of life. Liver Transpl Surg 1997;3:245-50.

37. Pageaux GP, Michel J, Coste V, et al. Alcoholic cirrhosis is a good indication for liver transplantation, even for cases of recidivism. Gut 1999;45:421-6.

38. Jain AB, Yee LD, Nalesnik MA, et al. Comparative incidence of de novo nonlymphoid malignancies after liver transplantation under tacrolimus using surveillance epidemiologic end result data. Transplantation 1998;66:1193-200.

39. Jonas S, Rayes N, Neumann U, et al. De novo malignancies after liver transplantation using tacrolimus-based protocols or cyclosporine-based quadruple immunosuppression with an interleukin-2 receptor antibody or antithymocyte globulin. Cancer 1997;80:1141-50.

40. Sanchez EQ, Marubashi S, Jung G, et al. De novo tumors after liver transplantation: a single-institution experience. Liver Transpl 2002;8:285-91.

41. Frezza EE, Fung JJ, van Thiel DH. Non-lymphoid cancer after liver transplantation. Hepatogastroenterology 1997;44:1172-81.

42. Thun MJ, Peto R, Lopez AD, et al. Alcohol consumption and mortality among middle-aged and elderly U.S. adults. N Engl J Med 1997;337:1705-14.

43. Duvoux C, Delacroix I, Richardet JP, et al. Increased incidence of oropharyngeal squamous cell carcinomas after liver transplantation for alcoholic cirrhosis. Transplantation 1999;67:418-21.

44. Tallón Aguilar L, Barrera Pulido L, Bernal Bellido C, et al. Causes and predisposing factors of de novo tumors in our series of liver transplant recipients. Transplant Proc 2009;41:2453-4.

45. Schmilovitz-Weiss H, Mor E, Sulkes J, et al. De novo tumors after liver transplantation: a single-center experience. Transplant Proc 2003;35:665-6.

46. Adami J, Gäbel H, Lindelöf B, et al. Cancer risk following organ transplantation: a nationwide cohort study in Sweden. Br J Cancer 2003;89:1221-7.

47. Presser SJ, Schumacher G, Neuhaus R, et al. De novo esophageal neoplasia after liver transplantation. Liver Transpl 2007;13:443-50.

48. Gaglio PJ, Gaglio PJ. Complications in patients with alcohol-associated liver disease who undergo liver transplantation. Clin Liver Dis 2012;16:865-75. 
49. Sint Nicolaas J, de Jonge V, Steyerberg EW, et al. Risk of colorectal carcinoma in post-liver transplant patients: a systematic review and meta-analysis. Am J Transplant 2010;10:868-76.

50. Vera A, Gunson BK, Ussatoff V, et al. Colorectal cancer in patients with inflammatory bowel disease after liver transplantation for primary sclerosing cholangitis. Transplantation 2003;75:1983-8.

51. Bleday R, Lee E, Jessurun J, et al. Increased risk of early colorectal neoplasms after hepatic transplant in patients with inflammatory bowel disease. Dis Colon Rectum 1993;36:908-12.

52. Fabia R, Levy MF, Testa G, et al. Colon carcinoma in patients undergoing liver transplantation. Am J Surg 1998;176:265-9.

53. Sérée O, Altieri M, Guillaume E, et al. Longterm risk of solid organ de novo malignancies after liver transplantation: a French national study on 11,226 patients. Liver Transpl 2018;24:1425-36.

54. Jiménez C, Marqués E, Loinaz C, et al. Upper aerodigestive tract and lung tumors after liver transplantation. Transplant Proc 2003;35:1900-1.

55. Jiménez C, Marqués $\mathrm{E}$, Manrique A, et al. Incidence and risk factors of development of lung tumors after liver

doi: $10.21037 / \operatorname{tgh} .2019 .11 .18$

Cite this article as: Singh A, De A, Singh V. Post-transplant malignancies in alcoholic liver disease. Transl Gastroenterol Hepatol 2020;5:30. transplantation. Transplant Proc 2005;37:3970-2.

56. Jiménez C, Manrique A, Marqués E, et al. Incidence and risk factors for the development of lung tumors after liver transplantation. Transpl Int 2007;20:57-63.

57. Finkenstedt A, Graziadei IW, Oberaigner W, et al. Extensive surveillance promotes early diagnosis and improved survival of de novo malignancies in liver transplant recipients. Am J Transplant 2009;9:2355-61.

58. Herrero JI, Bastarrika G, D’Avola D, et al. Lung cancer screening with low-radiation dose computed tomography after liver transplantation. Ann Transplant 2013;18:587-92.

59. Herrero JI, Alegre F, Quiroga J, et al. Usefulness of a program of neoplasia surveillance in liver transplantation. A preliminary report. Clin Transplant 2009;23:532-6.

60. Rademacher S, Seehofer D, Eurich D, et al. The 28year incidence of de novo malignancies after liver transplantation: a single-center analysis of risk factors and mortality in 1616 patients. Liver Transpl 2017;23:1404-14.

61. Lucey MR, Terrault N, Ojo L, et al. Long-term management of the successful adult liver transplant: 2012 practice guideline by the American Association for the Study of Liver Diseases and the American Society of Transplantation. Liver Transpl 2013;19:3-26. 